\title{
Representing Social Media Users for Sarcasm Detection
}

\author{
Y. Alex Kolchinski \\ Stanford University \\ kolchinskiestanford. edu
}

\author{
Christopher Potts \\ Stanford University \\ cgpottsestanford.edu
}

\begin{abstract}
We explore two methods for representing authors in the context of textual sarcasm detection: a Bayesian approach that directly represents authors' propensities to be sarcastic, and a dense embedding approach that can learn interactions between the author and the text. Using the SARC dataset of Reddit comments, we show that augmenting a bidirectional RNN with these representations improves performance; the Bayesian approach suffices in homogeneous contexts, whereas the added power of the dense embeddings proves valuable in more diverse ones.
\end{abstract}

\section{Introduction}

Irony and sarcasm ${ }^{1}$ are extreme examples of context-dependence in language. Given only the text Great idea! or What a hardship!, we cannot resolve the speaker's intentions unless we have insight into the circumstances of utterance - who is speaking, and to whom, and how the content relates to the preceding discourse (Clark, 1996). While certain texts are biased in favor of sarcastic uses (Kreuz and Caucci, 2007; Wallace et al., 2014), the non-literal nature of this phenomenon ensures that there is an important role for pragmatic inference (Clark and Gerrig, 1984).

The current paper is an in-depth study of one important aspect of the context dependence of sarcasm: the author. Our guiding hypotheses are that authors vary in their propensity for using sarcasm, that this propensity is influenced by more general facts about the context, and that authors have their own particular ways of indicating sarcasm. These hypotheses are well supported by psycholinguistic research (Colston and Lee, 2004; Gibbs, 2000; Dress et al., 2008), but our ability to test them

\footnotetext{
${ }^{1}$ We use "sarcasm" to include both sarcasm and irony, as the two are generally conflated in the literature we review.
}

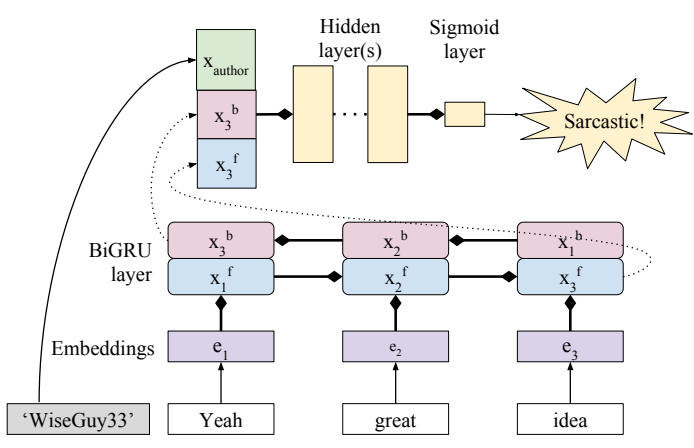

Figure 1: The model architecture. Look-ups are indicated by arrows, dense connections by diamonds. The author embedding can be null (a text-only baseline), a prior reflecting the author's propensity for sarcasm, or a learned embedding. There are potentially multiple layers between the initial example embedding and the output sigmoid layer.

at scale has until recently been limited by available annotated corpora. With the release of the Self-Annotated Reddit Corpus (SARC), Khodak et al. (2017) have helped to address this limitation. SARC is large and diverse, and its distribution of users across comments and forums makes it particularly well suited to modeling authors and their relationship to sarcasm.

Our core model of comment texts is a bidirectional RNN with GRU cells. To model authors, we propose two strategies for augmenting these RNN representations: a simple Bayesian method that captures only an author's raw propensity for sarcasm, and a dense embedding method that allows for complex interactions between author and text (Figure 1). We find that, on SARC, the simple Bayesian approach does remarkably well, especially in smaller, more focused forums. On the full SARC dataset, author embeddings are able to encode more kinds of variation and interaction with the text, and thus they achieve the highest predictive accuracy. These findings extend and reinforce 
the prior work on user-level modeling for sarcasm (Section 2), and they indicate that simple representation methods are effective here.

\section{Previous Work}

A substantial literature exists around sarcasm detection. Many of the prior studies focus on the analysis of Twitter posts, which lend themselves well to sarcasm detection with NLP methods because they are available in large quantities, they tend to correspond roughly to a single utterance, and users' hashtags in tweets (e.g., \#sarcasm, \#not) can provide imperfect but useful labels. A central theme of this literature is that bringing in contextual features helps performance.

González-Ibánez et al. (2011) trained classifiers using a combination of lexical and pragmatic features, including emoticons and whether the user was responding to another tweet (see also Felbo et al. 2017). Bamman and Smith (2015) extend this kind of analysis with additional information about the context. Of special interest here are their contextual features: the author's historical sentiment, topics, and terms; the addressee; and features drawn from historical interactions between the author and addressee. The study finds most features to be useful, but a model trained on the tweet and author features alone achieved essentially the same performance ( $84.9 \%$ accuracy) as a model trained on all features $(85.1 \%)$.

In a similar vein, Rajadesingan et al. (2015) used a complex combination of features from users' Twitter histories, including sentiment, grammar, and word choice, as inputs into their model, and report a $\approx 7 \%$ gain in classification accuracy upon adding these features to a baseline $n$ gram classifier.

Recent papers have also applied deep learning methods to detecting sarcastic tweets. Poria et al. (2016) use a combination convolutional-SVM architecture with auxiliary sentiment input features. The architecture of Zhang et al. (2016) includes an $\mathrm{RNN}$, and uses contextual features as well as tweet text for inputs.

Amir et al. (2016) extend the work of Bamman and Smith by generating author embeddings to reflect users' word-usage patterns (but not sarcasm history) in a manner similar to the paragraph vectors introduced by Le and Mikolov (2014). With the inclusion of these embeddings, their convolutional neural network (CNN) achieves a $2 \%$ gain in accuracy over that of Bamman and Smith.

Ghosh and Veale (2017) present a combination CNN/LSTM (long short-term memory RNN) architecture that takes as inputs user affect inferred from recent tweets as well as the text of the tweet and that of the parent tweet. When a tweet was addressed to someone by name, the name of the addressee was included in the text representation of the tweet, providing a loose link between interlocutors (West et al., 2014) and a $\approx 1 \%$ gain in performance for some data sets.

There has also been a small amount of previous work on Reddit data for sarcasm (Tay et al., 2018; Ghosh and Muresan, 2018). Wallace et al. (2014) explore a hand-labeled dataset of $\approx 3 \mathrm{~K}$ Reddit comments from six subreddits. They report that, when human graders attempted to mark comments as sarcastic or not sarcastic, they needed additional context like subreddit norms and author history roughly $30 \%$ of the time, and that the comments which graders found ambiguous were largely the same as those on which a baseline bagof-words classifier tended to make mistakes. In a follow-up study, Wallace et al. (2015) find that semantic cues for sarcasm differ by subreddit, and they show classifier accuracy gains when modeling subreddit-specific variation.

The work that is closest to our own is that of Hazarika et al. (2018), who also experiment on the SARC dataset. Their model learns author, forum, and text embeddings, and they show that all three kinds of representation contribute positively to the overall performance. We take a much simpler approach to author embeddings and do not include forum embeddings, and we report comparable performance (Section 6). We take this as further indication of the value of author features for modeling sarcasm.

\section{The SARC Dataset}

The Self-Annotated Reddit Corpus (SARC) was created by Khodak et al. (2017). ${ }^{2}$ It includes an unprecedented 533M comments. The corpus is self-annotated in the sense that a comment is considered sarcastic if its author marked it with the "/s" tag. As a result, the positive examples are essentially those which the authors considered ambiguous enough to explicitly tag as sarcastic, meaning that the prediction problem is actually to identify which comments are not only sarcastic but

\footnotetext{
${ }^{2}$ http://nlp.cs.princeton.edu/SARC/2.0/
} 


\begin{tabular}{lrr}
\hline & Comments & \% sarcastic \\
\hline Entire corpus (bal.) & 257,082 & 50.00 \\
r/politics (bal.) & 13,668 & 50.00 \\
r/politics (unbal.) & 309,925 & 3.06 \\
r/AskReddit (bal.) & 11,660 & 50.00 \\
r/AskReddit (unbal) & $1,548,803$ & 0.53 \\
\hline
\end{tabular}

Table 1: Basic statistics for SARC.

both sarcastic and not obviously so.

The dataset is filtered in numerous ways, and has good precision (only $\approx 1 \%$ false positive rate) but poor recall ( $2 \%$ false negatives relative to $0.25 \%$ true positives, or $\approx 11 \%$ recall). To alleviate the issues caused by low recall, the dataset also includes a balanced sample, where comments are supplied in pairs, both responding to the same parent comment and with exactly one of the two tagged as sarcastic. All comments are accompanied with ancestor comments from the original conversation, author information, and a score as voted on by Reddit users.

This dataset presents numerous advantages for sarcasm detection. For one, it is vastly larger than past sarcasm datasets, which enables the training of more sophisticated models. In addition, most work in sarcasm detection has focused on tweets, which are very short and tend to use abbreviated and atypical language. Reddit comments are not constrained by length and are therefore more representative of how people typically write. Finally, Reddit is organized into topically-defined communities known as subreddits, each of which has its own community norms and linguistic patterns. By making available large amounts of data from a number of subreddits, SARC facilitates the comparative analysis of subreddits, and more generally provides a view into the differences between communities.

Table 1 provides basic statistics on the entire corpus as well as the subreddits that we focus on in our experiments.

\section{Models}

Our baseline model is a bidirectional RNN with GRU cells (BiGRU; Cho et al. 2014). We tried variants with LSTM cells and did not observe a significant difference in performance. We therefore chose to use GRU cells as the model with fewer parameters. ${ }^{3}$

The inputs to the BiGRU model are users' comments, which are split into words (and in the case of conjunctions, subwords) and punctuation marks and are converted to word vectors. The final states of the two directions of the BiGRU are concatenated with each other and run through either a single fully-connected linear layer or two fullyconnected linear layers with a rectified linear unit in between. The output of the final linear layer is fed through a sigmoid function which outputs the estimated probability of sarcasm. This baseline does not take author information into account: for each comment, only the words of the comment are considered as inputs.

The Bayesian prior model extends the BiGRU with the sarcastic and non-sarcastic comment counts for authors seen in the training data, which serves as a prior for sarcasm frequency. This version of the model takes as inputs both a representation of the comment and the author representation $x_{\text {author }} \in \mathbb{Z}_{\geq 0}^{2}$ to estimate the probability of sarcasm. The model can be interpreted as computing a posterior probability of sarcasm given both the comment and the prior of previous sarcastic and non-sarcastic comment counts - author modeling reduced to a Bernoulli prior. For previously unseen authors, $x_{\text {author }}$ is set to $(0,0)$.

The author embedding approach extends the baseline BiGRU in a more sophisticated way. Here, each author seen in the training data is associated with a randomly initialized embedding vector $x_{\text {author }} \in \mathbb{R}^{15}$, which is then provided as an input to the model along with a representation of the words of the comment. A special randomly initialized vector $x_{\mathrm{UNK}}$ is used for previously unseen authors. The author embeddings are updated during training, with the goal of learning more sophisticated individualized patterns of sarcasm than the Bayesian prior allows. We experimented with training the $x_{\mathrm{UNK}}$ vector on infrequently-seen authors (fewer than 5 comments in the training set) instead of using a random vector, and found some suggestions of improved performance. However, as the differences in performance were not substantial enough to change the relative performance of the different models, we report the results for the simpler random- $x_{\mathrm{UNK}}$ model.

\footnotetext{
${ }^{3}$ Our models and associated experiment code are available at https://github.com/kolchinski/ reddit-sarc
} 


\section{Experiments}

We conducted three sets of experiments, one for each model, to evaluate the effectiveness of the different approaches to author modeling. Each set of experiments was conducted on five datasets: the balanced version of the entire corpus as well as the balanced and unbalanced versions of the r/politics and $\mathrm{r} /$ AskReddit subcorpora (Table 1).

In all cases, the raw comment data was tokenized into words and punctuation marks, with components of contractions treated as individual words. We mapped tokens to FastText embedding vectors which had been trained, using subword infomation, on Wikipedia 2017, the UMBC webbase corpus, and the statmt.org news dataset (Mikolov et al., 2018). While vectors existed for nearly $100 \%$ of tokens generated, exceptions were mapped to a randomly initialized UNK vector.

All models were trained with early stopping on a randomly partitioned holdout set of either $5 \%$ of the data for balanced subreddit corpora or $1 \%$ for the others. The performance of the model, as used for hyperparameter tuning, was evaluated against a second holdout set, generated in the same manner as the first holdout set but disjoint from both it and the portion of the data used for training.

Hyperparameters were tuned to maximize model performance as evaluated in this manner, starting with a randomized search process and fine-tuned manually. The final evaluation was conducted against the test set, with a single randomly partitioned holdout set from the training data again used for early stopping. We applied dropout (Srivastava et al., 2014) during training before and between all linear layers. For additional regularization, we also applied an 12-norm penalty to the linear weights but not to the GRU weights.

We attempted other model variations, including multiple GRU layers and an attention mechanism for GRU outputs, but did not observe any gains in performance from the larger models.

\section{Results and Discussion}

\subsection{Quantitive assessment}

Table 2 reports the means of 10 runs to control for variation deriving from randomness in the optimization process (Reimers and Gurevych, 2017).

Where there is overlap between our experiments and those of Hazarika et al. (2018) (CASCADE), our model is highly competitive. We slightly under-perform on the full balanced dataset but come out ahead on $\mathrm{r} /$ politics. This is striking because our model makes use of much less information. First, unlike CASCADE, we do not have forum embeddings. Second, CASCADE author embeddings involve extensive feature engineering including "stylometric" and "personality" features. Our author embeddings, on the other hand, are either simple empirical estimates (Bayesian priors) or learned embeddings with random initializations, in both cases allowing simpler model specification and training, and more flexibility on the task for which they are used.

There is also evidence that the BiGRU yields better representations of texts than does Hazarika et al.'s CNN-based model. Our 'No embed' model is akin to their CASCADE with no contextual features, which achieves only 0.66 on the full balanced corpus and 0.70 on the r/politics balanced dataset. Both numbers are well behind our 'No embed'. Unfortunately, we do not have space for a fuller study of the similarities and differences between our model and CASCADE.

Both of our methods for representing authors perform well. This is perhaps especially striking for the unbalanced experiments, where the percentage of sarcastic comments is tiny (Table 1). The two methods perform differently on individual forums than on the full dataset. For the $r$ /politics and r/AskReddit communities, the Bayesian priors give the best results. The situation is reversed for the full dataset, where the highdimensional embeddings outperform the Bayesian priors. This likely reflects two interacting factors. First, with smaller, more focused forums, it is harder to learn good author embeddings, so the simple prior is more reliable. Second, on the full dataset, there are more examples, and also more complex interactions between authors and their texts, so the added representational power of the embeddings proves justified.

\subsection{Qualitative comparisons}

Table 3 provides example predictions from the different models. Each example is taken from the holdout set of a run in which all three models were trained on the same training set and evaluation was conducted on the same holdout set.

For both sarcastic and non-sarcastic comments, author modeling can be helpful for disambiguation. For instance, in examples 1 and 2, omitting 


\begin{tabular}{llllll}
\hline & & \multicolumn{2}{c}{ r/politics } & \multicolumn{2}{c}{ r/AskReddit } \\
& Full balanced & \multicolumn{1}{c}{ balanced } & unbalanced & balanced & unbalanced \\
\hline No embed & $74.8[74.6,74.9]$ & $74.3[74.1,74.6]$ & $58.7[58.2,59.1]$ & $64.3[63.4,65.2]$ & $56.9[56.6,57.2]$ \\
Bayesian prior & $74.0[73.7,74.3]$ & $77.6[77.4,77.9]$ & $64.7[64.6,64.8]$ & $69.1[68.8,69.4]$ & $57.7[57.6,57.7]$ \\
15d embed & $75.3[74.8,75.7]$ & $75.1[74.4,75.8]$ & $62.0[59.9,63.9]$ & $66.0[65.1,66.8]$ & $57.1[56.6,57.6]$ \\
Khodak et al. (2017) & 75.8 & 76.5 & 27.0 & - & - \\
CASCADE & 77.0 & 75.0 & - & - & - \\
\hline
\end{tabular}

Table 2: Mean macro-averaged F1 scores with bootstrapped 95\% confidence intervals, based on 10 runs. CASCADE is the best system of Hazarika et al. (2018), and we report the strongest baseline numbers established by Khodak et al. (2017).

\begin{tabular}{lcccc}
\hline & \multicolumn{4}{c}{ Model Predictions of p(sarcastic) } \\
\multicolumn{1}{l}{ Reddit comment } & Sarcastic? & No user rep. & Bayesian & Multidimensional \\
\hline $\begin{array}{l}\text { 1. Good thing Trump is going to bring } \\
\text { back all those low education high pay- }\end{array}$ & Yes & .45 & .68 & .84 \\
ing jobs. & & & & \\
\hline 2. lol woops! & No & .78 & .36 & .25 \\
\hline $\begin{array}{l}\text { 3. The most ubiquitous undergarments I } \\
\text { see these days. }\end{array}$ & Yes & .15 & .17 & .79 \\
\hline $\begin{array}{l}\text { 4. Such a deep confession, and it doesn't } \\
\text { sound like the guy who wrote it is an } \\
\text { asshole at all. }\end{array}$ & Yes & .33 & .45 & .86 \\
\hline $\begin{array}{l}\text { 5. It's not entirely impossible that there } \\
\text { are recipe's that have yet to be discov- } \\
\text { ered. }\end{array}$ & No & .23 & .23 & .81 \\
\hline
\end{tabular}

Table 3: Examples selected to highlight differences between the models.

author modeling led to incorrect predictions, but including the frequency of the author's sarcasm use alone was enough to change the prediction from incorrect to correct.

In cases like examples 3 and 4, where the Bayesian prior was insufficient, including a model of the author's individualized patterns of sarcasm was much more powerful. That said, the more complex embedding model can misfire, as in example 5, where the simpler models make a correct prediction but it does not. This appeared to happen more for non-sarcastic examples, where the embedding model would occasionally strongly influence the predicted probability of sarcasm upward. Evidently, authors have more individualized patterns of sarcasm than of non-sarcasm.

Judging by the relative performance of the Bayesian and multidimensional-embedding models (Table 2), the multidimensional model wins more disagreements than it loses with the
Bayesian model when there is more training data available. However, when there is not, it overfits to such a degree that its predictions of authors' sarcasm patterns are less useful than the Bayesian approach. This suggests a future direction of exploration: the most useful model of all may be one that expands in complexity for authors with more examples available, and shrinks for those who have fewer.

\section{Conclusion}

This paper evaluated two data-driven methods for modeling the role of the author in sarcasm detection. Both prove effective. As shown by Hazarika et al. (2018), similar techniques can be extended to other aspects of the context. While our experiments did not support adding these representations, we think listeners rely on them as well, so additional computational modeling work here is likely to prove fruitful. 


\section{References}

Silvio Amir, Byron C Wallace, Hao Lyu, and Paula Carvalho Mário J Silva. 2016. Modelling context with user embeddings for sarcasm detection in social media. arXiv preprint arXiv:1607.00976.

David Bamman and Noah A Smith. 2015. Contextualized sarcasm detection on twitter. In ICWSM, pages 574-577.

Kyunghyun Cho, Bart van Merrienboer, Caglar Gulcehre, Dzmitry Bahdanau, Fethi Bougares, Holger Schwenk, and Yoshua Bengio. 2014. Learning phrase representations using rnn encoder-decoder for statistical machine translation. In Proceedings of the 2014 Conference on Empirical Methods in Natural Language Processing (EMNLP), pages 17241734. Association for Computational Linguistics.

Herbert H. Clark. 1996. Using Language. 'Using' Linguistic Books. Cambridge University Press.

Herbert H. Clark and Richard J. Gerrig. 1984. On the pretense theory of irony. Journal of Experimental Psychology: General, 113(1):121-126.

Herbert L. Colston and Sabrina Y. Lee. 2004. Gender differences in verbal irony use. Metaphor and Symbol, 19(4):289-306.

Megan L. Dress, Roger J. Kreuz, Kristen E. Link, and Gina M. Caucci. 2008. Regional variation in the use of sarcasm. Journal of Language and Social Psychology, 27(1):71-85.

Bjarke Felbo, Alan Mislove, Anders Søgaard, Iyad Rahwan, and Sune Lehmann. 2017. Using millions of emoji occurrences to learn any-domain representations for detecting sentiment, emotion and sarcasm. arXiv preprint arXiv:1708.00524.

Aniruddha Ghosh and Tony Veale. 2017. Magnets for sarcasm: Making sarcasm detection timely, contextual and very personal. In Proceedings of the 2017 Conference on Empirical Methods in Natural Language Processing, pages 482-491.

Debanjan Ghosh and Smaranda Muresan. 2018. " with 1 follower i must be awesome: P”. exploring the role of irony markers in irony recognition. arXiv preprint arXiv:1804.05253.

Raymond W. Gibbs. 2000. Irony in talk among friends. Metaphor and Symbol, 15(1-2):5-27.

Roberto González-Ibánez, Smaranda Muresan, and Nina Wacholder. 2011. Identifying sarcasm in twitter: a closer look. In Proceedings of the 49th Annual Meeting of the Association for Computational Linguistics: Human Language Technologies: Short Papers-Volume 2, pages 581-586. Association for Computational Linguistics.
Devamanyu Hazarika, Soujanya Poria, Sruthi Gorantla, Erik Cambria, Roger Zimmermann, and Rada Mihalcea. 2018. CASCADE: Contextual sarcasm detection in online discussion forums. ArXiv:1805.06413.

Mikhail Khodak, Nikunj Saunshi, and Kiran Vodrahalli. 2017. A large self-annotated corpus for sarcasm. arXiv preprint arXiv:1704.05579.

Roger J Kreuz and Gina M Caucci. 2007. Lexical influences on the perception of sarcasm. In Proceedings of the Workshop on computational approaches to Figurative Language, pages 1-4. Association for Computational Linguistics.

Quoc Le and Tomas Mikolov. 2014. Distributed representations of sentences and documents. In International Conference on Machine Learning, pages 1188-1196.

Tomas Mikolov, Edouard Grave, Piotr Bojanowski, Christian Puhrsch, and Armand Joulin. 2018. Advances in pre-training distributed word representations. In Proceedings of the International Conference on Language Resources and Evaluation (LREC 2018).

Soujanya Poria, Erik Cambria, Devamanyu Hazarika, and Prateek Vij. 2016. A deeper look into sarcastic tweets using deep convolutional neural networks. arXiv preprint arXiv: 1610.08815.

Ashwin Rajadesingan, Reza Zafarani, and Huan Liu. 2015. Sarcasm detection on twitter: A behavioral modeling approach. In Proceedings of the Eighth ACM International Conference on Web Search and Data Mining, pages 97-106. ACM.

Nils Reimers and Iryna Gurevych. 2017. Reporting score distributions makes a difference: Performance study of LSTM-networks for sequence tagging. ArXiv:1707.09861.

Nitish Srivastava, Geoffrey Hinton, Alex Krizhevsky, Ilya Sutskever, and Ruslan Salakhutdinov. 2014. Dropout: A simple way to prevent neural networks from overfitting. The Journal of Machine Learning Research, 15(1):1929-1958.

Yi Tay, Luu Anh Tuan, Siu Cheung Hui, and Jian Su. 2018. Reasoning with sarcasm by reading inbetween. arXiv preprint arXiv:1805.02856.

Byron C Wallace, Eugene Charniak, et al. 2015. Sparse, contextually informed models for irony detection: Exploiting user communities, entities and sentiment. In Proceedings of the 53rd Annual Meeting of the Association for Computational Linguistics and the 7th International Joint Conference on Natural Language Processing (Volume 1: Long Papers), volume 1, pages 1035-1044.

Byron C Wallace, Laura Kertz, Eugene Charniak, et al. 2014. Humans require context to infer ironic intent (so computers probably do, too). In Proceedings of the 52nd Annual Meeting of the Association 
for Computational Linguistics (Volume 2: Short Papers), volume 2, pages 512-516.

Robert West, Hristo S. Paskov, Jure Leskovec, and Christopher Potts. 2014. Exploiting social network structure for person-to-person sentiment analysis. Transactions of the Association for Computational Linguistics, 2(2):297-310.

Meishan Zhang, Yue Zhang, and Guohong Fu. 2016. Tweet sarcasm detection using deep neural network. In Proceedings of COLING 2016, the 26th International Conference on Computational Linguistics: Technical Papers, pages 2449-2460. 